\title{
MTHFR, MTR, and MTRR Polymorphisms in Relation to pl6INK4A Hypermethylation in Mucosa of Patients with Colorectal Cancer
}

\author{
Yvonne Wettergren, Elisabeth Odin, Göran Carlsson, and Bengt Gustavsson \\ Department of General Surgery, University of Gothenburg, Sahlgrenska University Hospital/Östra, Gothenburg, Sweden
}

\begin{abstract}
We recently analyzed the hypermethylation status of the pl6INK4a (p16) gene promoter in normal-appearing mucosa obtained from patients with colorectal cancer. Hypermethylation of p16 was associated with reduced survival of these patients. In the present study, germ line polymorphisms in the folate- and methyl-associated genes, methylenetetrahydrofolate reductase (MTHFR), methionine synthase (MTR) and methionine synthase reductase (MTRR), were analyzed in the same patient cohort to find a possible link between these genetic variants and p16 hypermethylation. Genomic DNA was extracted from blood of patients $(n=181)$ and controls $(n=300)$. Genotype analyses were run on an ABI PRISM ${ }^{\circledR} 7900 H T$ sequence-detection system (Applied Biosystems), using real-time polymerase chain reaction and TaqMan chemistry. The results showed that the genotype distributions of the patient and control groups were similar. No significant differences in cancer-specific or disease-free survival of stage I-III patients according to polymorphic variants were detected, nor were any differences in cancer-specific or disease-free survival detected when patients were subgrouped according to the MTHFR or MTR genotype groups and dichotomized by pl6 hypermethylation status in mucosa. However, patients with the MTRR 66 AA/AG genotypes were found to have a significantly worse cancer-specific survival when the mucosa were positive, compared with negative, for p 16 hypermethylation (hazard ratio $2.7 ; 95 \%$ confidence interval 1.2-6.4; $P=0.023$ ). In contrast, there was no difference in survival among patients with the MTRR 66 GG genotype stratified by 16 hypermethylation status. These results indicate a relationship between genetic germ-line variants of the MTRR gene and pl6 hypermethylation in mucosa, which may affect the clinical outcome of patients with colorectal cancer.
\end{abstract}

(C) 2010 The Feinstein Institute for Medical Research, www.feinsteininstitute.org

Online address: http://www.molmed.org

doi: $10.2119 /$ molmed.2009.00156

\section{INTRODUCTION}

The tumor-suppressor gene $p 16$ codes for a cyclin-dependent kinase inhibitor, p16, that acts like a negative regulator of cell growth and proliferation in the G1 phase of the cell cycle (1). In addition, recent research has implicated p16 as an important regulator of the angiogenic switch (2). In a previous study (3), we analyzed the hypermethylation status of the $p 16$ gene promoter in macroscopically normal-appearing mucosa derived from 181 patients with colorectal cancer (CRC). The result showed that hypermethylation of $p 16$ was associated with re- duced survival of the patients. In mucosa samples without hypermethylation of p16 there was a strong correlation between gene expression of reduced folate carrier 1 and folylpolyglutamate synthase (FPGS, EC 6.3.2.17), possibly indicating a balance between uptake and retention of folates. No such correlation was found in mucosa samples displaying p16 hypermethylation.

Within the cells, folate polyglutamates are converted to 5,10-methylenetetrahydrofolate (methylene-THF), which is required as a methyl donor in the synthesis of dTMP (2'-deoxythymidine-

Address correspondence and reprint requests to Yvonne Wettergren, Surgical-Oncology Laboratory, Department of General Surgery, University of Gothenburg, Plan 1, CK, Sahlgrenska University Hospital/Östra, S-416 85 Gothenburg, Sweden. Phone: 46-31-3434968; Fax: 46-31-343-5930; E-mail: yvonne.wettergren@dep-surg.gu.se. Submitted October 26, 2009; Accepted for publication June 10, 2010; Epub (www.molmed.org) ahead of print June 11, 2010.

5'-monophosphate) from dUMP (2'-deoxyuridine-5'-monophosphate) (Figure 1). Methylene-THF is also the precursor of metabolically active 5-methyltetrahydrofolate (methyl-THF), which is used in the remethylation of the sulfur-containing amino acid homocysteine to methionine. Endogenous methionine is then catabolized to produce the universal methyl donor S-adenosylmethionine (SAM). The conversion of methylene-THF to methylTHF is dependent on the enzyme methylenetetrahydrofolate reductase (MTHFR; EC 1.5.1.20). It is known that the MTHFR variant C677T causes reduced enzyme activity that impairs the folate metabolism, leading to genomic DNA hypomethylation (4) concomitant with gene-specific promoter hypermethylation (5), as is frequently observed in cancer. In addition, reduced MTHFR activity leads to increased plasma levels of 


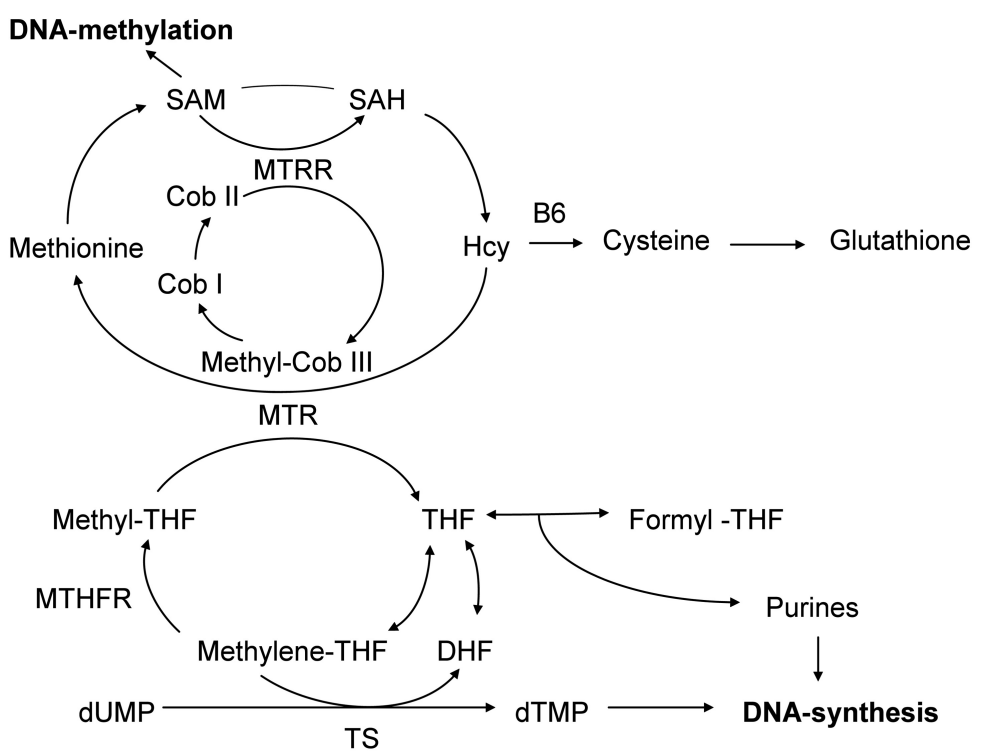

Figure 1. Overview showing the action of the MTHFR, MTR and MTRR enzymes in the folate and methionine metabolism pathways. TS, thymidylate synthase; DHF, dihydrofolate; Formyl-THF, 10-formyltetrahydrofolate; Cob I, cob(I)alamin; Cob II, cob(II)alamin; MethylCob III, methyl cob(III)alamin; SAH, S-adenosylhomocysteine; Hcy, homocysteine; B6, vitamin B6.

homocysteine. Increased levels of homocysteine may promote inflammatory processes by generating reactive oxygen species (ROS) (6). Hence, the metabolite has a crucial role in cellular oxidative stress. Homocysteine may also affect hypo- and hypermethylation of DNA (7). Several case-control studies have shown that the MTHFR C677T polymorphism is protective against CRC (8-10), especially among individuals with a folate/methylsufficient diet $(11,12)$. However, contradictive results have been found (13-16). Evaluation of the MTHFR C677T polymorphism as a predictive marker for CRC patients treated with 5-fluorouracil (5-FU)-based chemotherapy points to a possible link between the MTHFR 677 T allele and tumor response (10,17-19). Studies have also shown that the polymorphism might be associated with 5-FU-related cytotoxicity $(19,20)$.

The MTR A2756G polymorphism leads to replacement of aspartic acid with glycine in the protein-binding region of methionine synthase (MTR; EC 2.1.1.13) (21). This substitution leads to a less effective enzyme that induces modest reduction of homocysteine $(22,23)$. Paz et al. (24) analyzed the association of MTHFR and MTR genetic variants in colorectal, breast and lung cancer patients with 5-methylcytosine content in the genome of the tumors and their normal counterparts, as well as the presence of CpG-island hypermethylation in tumorsuppressor genes, including $p 16$. Carriers of the MTHFR 677 T allele showed constitutive low levels of 5-methylcytosine in their genomes, and tumors in these patients did not achieve severe degrees of global hypomethylation. Tumors occurring in homozygous carriers of the MTR $2756 \mathrm{G}$ allele showed a low number of hypermethylated $\mathrm{CpG}$ islands of tumor-suppressor genes. Kang et al. (25) recently showed that in uterine cervical cancer patients with the heterozygous genotypes of MTHFR and MTR, the average number of methylated genes was lower than in patients with the common homozygous genotypes. Occasionally, MTR loses its activity through oxidation of the cofactor cob(I)alamin to cob(II)alamin. The methionine synthase reductase (MTRR; EC 2.1.1.135) enzyme then reactivates MTR in a reaction in which methyl(III)cobalamin is generated
(26). The MTRR A66G polymorphism leads to a substitution of isoleucine with methionine at codon 22 in the MTRR enzyme (27), resulting in a variant protein exhibiting a 4-fold lower activity compared with the wild-type protein in vivo (28). Thus, the MTRR $66 \mathrm{G}$ allele should decrease the availability of SAM by reducing the level of active MTR. As a result, DNA hypomethylation may be induced. The MTR 2756 GG genotype has been associated with an increased risk of CRC (29), whereas a reduced risk of colorectal adenoma recurrence has been observed in patients heterozygous for the MTRR A66G polymorphism (30). The effects of the MTR A2756G and MTRR A66G variants seem to be influenced by folate intake $(9,31)$.

The relationship between genetic polymorphisms, folate and the $p 16$ gene has been studied by a few research groups. Kraunz et al. (32) found that low dietary intake of folate was associated with $p 16$ methylation in tumors of head and neck squamous cell carcinoma patients, and that this relationship was modified by the MTHFR genotype. Kamiya et al. (33) examined the association between the MTHFR polymorphism and expression of $p 16$. Their results suggested that folate metabolism can affect carcinogenesis through the expression of $p 16$. The aim of the present study was to analyze the association of single-nucleotide polymorphisms (SNPs) in the folate- and methylassociated genes MTHFR, MTR and $M T R R$ to the $p 16$ promoter hypermethylation status in colorectal mucosa of patients with nonhereditary CRC. The aim was also to estimate the value of the interaction of p16 promoter methylation with the SNPs when predicting clinical outcome.

\section{MATERIALS AND METHODS}

\section{Patients and Study Design}

Blood and surgically resected specimens from normal-appearing mucosa were initially obtained from 181 patients with nonhereditary colorectal adenocarcinoma diagnosed at the Sahlgrenska 
University Hospital/Östra during the period between 1994 and 2004. However, because of noninformative genotyping results, 1 patient was omitted from the study, giving a total of 180 patients. The ethics committee of Göteborg University approved the study and informed consent was obtained from each of the patients. Tissue samples were obtained by surgical resection from areas approximately $10 \mathrm{~cm}$ from the primary tumors. All samples were snap frozen in liquid nitrogen immediately after surgical excision and stored at $-70^{\circ} \mathrm{C}$ until use. Surgical records were reviewed for patient sex and age, and pathological records were reviewed for tumor location, differentiation and stage. The mean age of the patients was $69 \pm 14$ years: $70 \pm 15$ years for the women and $68 \pm 12$ years for the men. The primary tumors were graded histopathologically by experienced pathologists as recommended by the World Health Organization (34). Of the primary carcinomas, 4 were highly, 114 were moderately and 62 were poorly differentiated; 70 tumors were of rectal origin and 110 were colonic; 63 of the colonic tumors were right-sided and 47 were left-sided. Malignant tumors were classified according to the TNM (tumornode-metastasis) staging system (35) into the pathological stages TNM I $(n=10)$, TNM II $(\mathrm{n}=71)$, TNM III $(\mathrm{n}=51)$ and TNM IV $(\mathrm{n}=48)$. Twenty-nine of the patients underwent presurgical radiotherapy, 26 patients were treated with adjuvant chemotherapy after surgery and 29 underwent palliative treatment. The vast majority of the patients (167 of 180) were subjected to elective surgery whereas 10 were subjected to acute surgery (no information was available for 3 of the patients). The follow-up time of the study was 5 years.

\section{Blood Donors}

The control group $(n=300)$ consisted of white, Swedish blood donors of both sexes from the same geographic area as the study patients. All samples were collected at random and informed consent was obtained from each of the donors.

\section{Determination of $p 16$ Hypermethylation Status}

The hypermethylation status of $p 16$ was determined using real-time polymerase chain reaction (PCR) as described previously (3).

\section{Genotyping of the MTHFR, MTR and MTRR Genes}

Genomic DNA was extracted from fresh-frozen blood using a QIAamp DNA Mini Kit according to the manufacturer instructions (Qiagen, Hilden, Germany). Genotype analyses of the genes MTHFR (rs1801133, assay no. C_8714009_10), MTR (rs1805087, assay no. C_12005959_10) and MTRR (rs1801394, assay no. C_3068176_10) were run on an ABI PRISM ${ }^{\circledR} 7900 \mathrm{HT}$ sequence-detection system (Applied Biosystems, Foster City, CA, USA) using real-time PCR and TaqMan chemistry. The SNP assays (Applied Biosystems) and the TaqMan PCR master mix (Applied Biosystems) were aliquoted into a 384-well plate using a liquidhandling Biomek FX robot (Beckman Coulter, San Diego, CA, USA). Reactions were characterized by comparing the threshold cycle $\left(\mathrm{C}_{\mathrm{T}}\right)$ values as described by the manufacturer. All genotype analyses were performed by the Göteborg Genomics Core Facility (Göteborg, Sweden).

\section{Statistics}

For the statistical analyses, the clinicopathological parameters were categorized as follows: tumor location as right-sided colon, left-sided colon or rectum; tumor stages as TNM stages I-IV; tumor differentiation grade as low/medium or high; patient treatment with adjuvant chemotherapy as yes or no. Cancer-specific survival time was calculated from the date of surgery to the last time of follow-up or to the date of death caused by cancer. Disease-free survival time was calculated from the date of surgery to the last time of follow-up or to the date of recurrence. The statistical significance of the difference in the survival groups was calcu- lated by using the Cox proportional hazard model. Data were analyzed by statistical modeling using the commercial software programs JMP (version 7.0; SAS, Cary, NC, USA) or SPSS (version 16.0) (Chicago, IL, USA). Statistical differences between groups were tested by using the Fisher exact test or the Pearson chi-square test as indicated in the tables. Statistical values of $P \leq 0.05$ were judged as significant.

\section{RESULTS}

\section{Distribution of Genotypes in CRC Patients and Healthy Controls}

To investigate whether the MTHFR C677T, MTR A2756G and MTRR A66G polymorphisms were predictive for CRC, we compared the genotype frequencies of patients with those of 300 healthy, white blood donors (Table 1). As shown in Table 1, the genotype distributions of the patient group were not significantly different from those of the control group. Furthermore, none of the clinical or pathological parameters (sex, age, tumor location, tumor differentiation grade or tumor stage) were associated with genotypes (data not shown). The MTHFR C677T, MTR A2756G and MTRR A66G genotype distributions were in HardyWeinberg equilibrium in both patients and controls.

\section{Association of $p 16$ Hypermethylation with the MTHFR, MTR and MTRR Genotypes}

Table 2 shows the result of the MTHFR C677T, MTR A2756G and MTRR A66G analyses for CRC patients dichotomized by $p 16$ hypermethylation status in mucosa. As shown, the MTHFR 677 TT homozygous genotype was more common among patients with hypermethylated p16 compared with patients without hypermethylation, 10 of $62(16.1 \%)$ and 8 of $113(7.1 \%)$, respectively, but the difference did not reach significance. No differences were found when the MTR and $M T R R$ genotype distributions were dichotomized according to p16 hypermethylation status. 
Table 1. Distribution of MTHFR, MTR and MTRR genotypes in CRC patients and controls.

\begin{tabular}{lccc}
\hline & $\begin{array}{c}\text { CRC } \\
\text { patients, } \\
\mathrm{n}(\%)^{\mathrm{a}}\end{array}$ & $\begin{array}{c}\text { Controls, } \\
\mathrm{n}(\%)^{\mathrm{b}}\end{array}$ & $P^{\mathrm{c}}$ \\
Genotype & $\begin{array}{ccc} \\
\text { MTHFR 677 }\end{array}$ & \\
CC & $81(46.3)$ & $167(55.9)$ & \\
CT & $76(43.4)$ & $107(35.8)$ & \\
TT & $18(10.3)$ & $25(8.4)$ & 0.13 \\
MTR 2756 & & & \\
AA & $112(63.6)$ & $193(64.8)$ & \\
AG & $55(31.3)$ & $92(30.9)$ & \\
GG & $9(5.1)$ & $13(4.3)$ & 0.92 \\
MTRR 66 & & & \\
AA & $22(12.4)$ & $50(16.7)$ & \\
AG & $94(53.1)$ & $152(50.8)$ & \\
GG & $61(34.5)$ & $97(32.4)$ & 0.45 \\
\hline
\end{tabular}

a Of 180 patient samples, 175, 176 and 177 were informative for the MTHFR, MTR, and MTRR polymorphisms, respectively. bOf 300 control samples, 299, 298 and 299 were informative for the MTHFR, MTR and MTRR polymorphisms, respectively.

${ }^{c} P$ by Pearson chi-square test.

MTHFR, MTR and MTRR Genotypes and Survival of Stage I-III Patients Dichotomized by 16 Hypermethylation in Mucosa

The clinical follow-up time of the patients was extended in the present study compared with the previous one, and at the time of this report all patients had been followed for at least 5 years. Because of this, the survival data were reanalyzed to examine whether p16 hypermethylation in mucosa was still a risk factor for cancer-specific and disease-free survival of patients with stage I-III disease. As before, we used a multivariate Cox regression analysis model adjusted for known risk factors such as sex, age, tumor stage, tumor differentiation grade and tumor location. The model was also adjusted for whether or not the patients had been treated with radiotherapy prior to surgery and / or adjuvant chemotherapy after surgery. The results showed that the $p 16$ hypermethylation status was prognostic (hazard ratio [HR] 2.6, 95\% confidence interval $[\mathrm{CI}] 1.2-5.7 ; P=$ 0.013] and HR 2.2, 95\% CI 1.1-4.4;
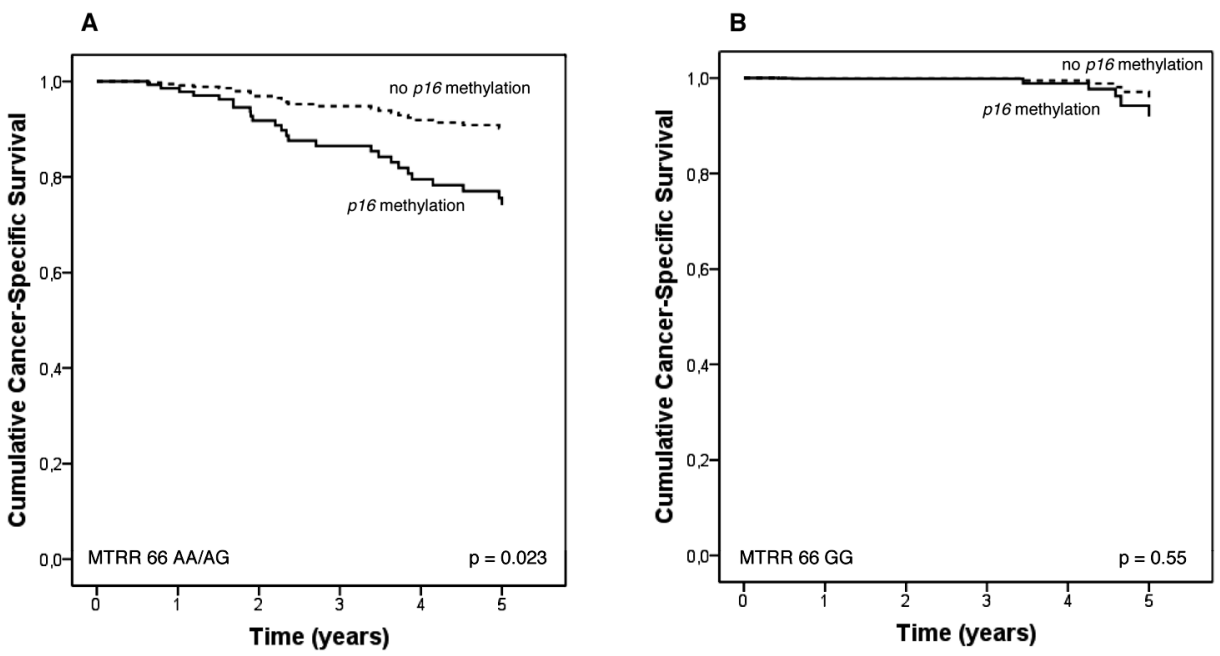

Figure 2. Multivariate Cox model showing cancer-specific survival of stage I-III patients subgrouped according to MTRR genotypes and dichotomized pl6 hypermethylation status in mucosa. (A) As shown, patients with the MTRR 66 AA/AG genotypes had a significantly worse survival $(P=0.023$ ) when the mucosa was positive for $p 16$ hypermethylation $(n=59)$ compared with when the mucosa was negative for $p 16$ hypermethylation $(n=$ 30). (B) In contrast, there was no difference in survival among patients with the MTRR 66 GG genotype stratified by pl6 hypermethylation status $(P=.55 ; n=24$ and $n=16$ in respective groups).

$P=0.033)$ for cancer-specific and disease-free survival, respectively.

Because of the low representation of individuals having the MTHFR 677 TT, MTR 2756 GG and MTRR 66 AA homozygous genotypes, each of these groups was pooled with the corresponding group of heterozygotes before survival analysis. No significant differences in cancer-specific or disease-free survival of stage I-III patients associated with the MTHFR, MTR and MTRR genotype variants could be detected. Nor were any differences in cancer-specific or disease-free survival detected when patients were subgrouped according to the MTHFR or $M T R$ genotype groups and dichotomized by $p 16$ hypermethylation status in mucosa. However, patients with the MTRR 66 AA / AG genotype had a significantly worse cancer-specific survival (HR 2.7, 95\% CI 1.2-6.4; $P=0.023$ ) (Figure 2A) and disease-free survival (HR $2.3,95 \% \mathrm{CI}$ $1.0-5.0 ; P=0.044)$ when the $p 16$ gene was hypermethylated in mucosa. As shown in Table 3, there was no difference in the distribution of patients having the MTRR AA/AG genotypes according to tumor stage, tumor differentiation grade or therapy conditions after dichotomization by $p 16$ hypermethylation status. No differences in cancer-specific (Figure 2B) or disease-free survival according to $p 16$ hypermethylation status were observed among patients having the MTRR 66 GG genotype.

\section{DISCUSSION}

The major mechanism of $p 16$ gene inactivation associated with development of CRC seems to be promoter-region methylation (36). Recently, Belshaw et al. (37) showed that multinomial logistic regression models based on the $\mathrm{CpG}$ island methylation profiles from normal mucosa could be used to distinguish cancer patients from noncancer patients and that p16 was one of the most informative variables. As previously reported by us (3), the hypermethylation status of the $p 16$ gene promoter in mucosa obtained $10 \mathrm{~cm}$ from the tumor site was associated with outcome of the CRC patients. In the present study, the same patient cohort was analyzed regarding the possible link between genetic germ-line polymorphisms 
Table 2. Distribution of MTHFR, MTR and MTRR genotypes in the CRC patients dichotomized by p16 hypermethylation status in mucosa.

\begin{tabular}{cccc}
\hline & $\begin{array}{c}\text { pl6 Hypermethylation } \\
\text { status }\end{array}$ & \\
\cline { 2 - 3 } Genotype & $\begin{array}{c}\text { Negative, } \\
\mathrm{n}(\%)\end{array}$ & $\begin{array}{c}\text { Positive, } \\
\mathrm{n}(\%)\end{array}$ & $P^{\mathrm{a}}$ \\
\hline MTHFR 677 & & & \\
CC & $56(49.6)$ & $25(40.3)$ & \\
CT & $49(43.4)$ & $27(43.6)$ & \\
TI & $8(7.0)$ & $10(16.1)$ & 0.14 \\
MTR 2756 & & & \\
AA & $66(59.5)$ & $46(70.8)$ & \\
AG & $39(35.1)$ & $16(24.6)$ & \\
GG & $6(5.4)$ & $3(4.6)$ & 0.31 \\
MTRR 66 & & & \\
AA & $15(13.3)$ & $7(10.9)$ & \\
AG & $58(51.3)$ & $36(56.3)$ & \\
GG & $40(35.4)$ & $21(32.8)$ & 0.80 \\
\hline
\end{tabular}

a $p$ by Pearson chi-square test.

of the genes MTHFR, MTR and MTRR and the hypermethylation status of $p 16$.

MTHFR, MTR and MTRR are important enzymes in the folate and methyl pathways and each enzyme has polymorphic variants that might be associated with risk of CRC. However, the data showed that the genotype distributions of these genes did not differ significantly between patient and control groups, indicating that none of the genetic variants had influenced the cancer susceptibility. There appeared to be no link between any of the polymorphic variants and the p16 methylation status, because no significant differences according to genotype distributions in patients subgrouped by $p 16$ hypermethylation status could be found. Because the clinical follow-up time of the patients was extended in the present study compared with the previous one, we reanalyzed the survival data. As before, $p 16$ hypermethylation was found to be an independent prognostic marker for cancerspecific survival. Analysis of the whole group of stage I-III patients showed that none of the genetic variants MTHFR C677T, MTR A2756G or MTRR A66G were related to cancer-specific or diseasefree survival. However, patients with the MTRR 66 AA / AG genotype had a signif-

Table 3. Distribution of patients having the MTRR 66 AA/AG genotypes according to tumor stage, tumor differentiation grade, and therapy conditions, after dichotomization by p16 hypermethylation status

\begin{tabular}{|c|c|c|c|}
\hline & $\begin{array}{c}\text { No p16 hypermethylation, } \\
\text { n (\%) }\end{array}$ & $\begin{array}{c}\text { pl6 Hypermethylation, } \\
\text { n (\%) }\end{array}$ & $P^{a}$ \\
\hline \multicolumn{4}{|l|}{ Tumor stage } \\
\hline $1+\|$ & $35(59)$ & $19(63)$ & \\
\hline III & $24(41)$ & $11(37)$ & 0.82 \\
\hline \multicolumn{4}{|c|}{ Tumor differentiation grade } \\
\hline Low/medium & $40(68)$ & $20(67)$ & \\
\hline High & $19(32)$ & $10(33)$ & 1.0 \\
\hline \multicolumn{4}{|c|}{ Adjuvant chemotherapy } \\
\hline No & $47(80)$ & $26(87)$ & \\
\hline Yes & $12(20)$ & $4(13)$ & 0.56 \\
\hline \multicolumn{4}{|c|}{ Presurgical radiotherapy } \\
\hline No & $49(83)$ & $25(83)$ & \\
\hline Yes & $10(17)$ & $5(17)$ & 1.0 \\
\hline
\end{tabular}

${ }^{a} p$ by Fisher exact test.

icantly worse cancer-specific survival if the $p 16$ gene was hypermethylated in mucosa. In contrast, there was no difference in survival related to $p 16$ hypermethylation among patients with the MTRR 66 GG genotype.

The reason for the particularly unfavorable outcome of patients having the combination of the MTRR 66 AA/AG genotype and $p 16$ hypermethylation in mucosa might be linked to a state of chronic inflammation and oxidative stress. Chen $e t$ al. found that a number of inflammation markers were altered in mucosa of patients with CRC compared with controls (38). These alterations could be found in mucosa several centimeters from the tumor site. It is known that a long-standing chronic inflammation in the colorectal epithelium will lead to oxidative stress (39), a condition considered to be one of the major sources of carcinogenesis. Growing evidence supports a role of ROS-induced generation of oxidative stress in epigenetic changes such as hypo- and hypermethylation (40). Interestingly, inactivation of $p 16$ by homozygous deletion or methylation at the promoter region seems to be a common molecular mechanism in oxidative stress-induced carcinogenesis $(41,42)$. Romanenko et al. (43) presented data suggesting that oxidative stress-induced methylation and inactivation of $p 16$ by persistent chronic exposure to ionizing radiation could be one of the major pathways responsible for malignant progression of renal cell carcinomas. Likewise, the results of Belinsky et al. (44) supported a possible role for oxidative stress, inflammation and $p 16$ methylation in the etiology of human lung cancer. The link between p16 and oxidative stress was also demonstrated by Govindarajan et al. (45), who analyzed the mechanisms behind the appearance of soft-tissue sarcomas at sites of foreign material implantations. Malignant fibrous histiocytomas arose in mice after implantation of nickel sulfide and all tumors showed hypermethylation of $p 16$. The authors concluded that the oxidative stress that was induced caused loss of p16. Jang et al. (46) showed that nontumorous tissue adjacent to gastric cancers displayed a close association among the grade of chronic inflammation and $p 16$ gene promoter hypermethylation.

During the state of chronic inflammation, DNA damage and mutations are induced by increased production of ROS and electrophiles. Consequently, the need for folates used in DNA synthesis and repair will be increased. Because folates are very susceptible to oxidation, an increased oxidative degradation of folates may become relevant under oxidative 
stress conditions. Thus, oxidative stress could represent an endogenous reason for folate deficiency, even when the dietary intake is within the recommended range (47). The relationship between promoter methylation of genes involved in colorectal carcinogenesis, including $p 16$, and folate was recently investigated (48). The results showed that low folate/high alcohol intake was associated with increased gene promoter hypermethylation in CRC. In our previously published study (3), FPGS gene expression (used as a surrogate marker for folate) was shown to be lower in mucosa with $p 16$ hypermethylation, compared with mucosa without it. The difference did not reach significance $(P=0.079)$, however, possibly due to the limited number of samples that could be analyzed.

Folates are needed in the elimination of homocysteine via both the transmethylation and the transsulfuration pathways (49). Under physiological conditions, the strongest risk factor for hyperhomocysteinemia seems to be low serum folate levels (7). In cases of increased oxidative stress, however, the homocysteine level may not reflect folate deficiency but rather a deficiency in vitamin $B_{12}$ (cobalamin) (50). Markedlyelevated concentrations of homocysteine have been found in colonic mucosa of inflammatory bowel disease patients $(51,52)$. In patients with Crohn disease the MTRR A66G polymorphism was shown to be associated with oxidant stress and disease activity, whereas vitamin $B_{12}$ and the MTHFR 677 TT genotype were the main determinants of hyperhomocysteinemia (53). The MTRR 66 AA genotype was a significant independent predictor of Crohn disease risk, and patients with this genotype had significantly higher levels of the oxidative stress marker, superoxide dismutase. Thus, it is possible that CRC patients having the MTRR 66 AA variant are more predisposed to oxidative stress.

Although the relatively small number of cases included in the present study leads to a limited statistical power, especially for subgroup analyses, the results indicate an association of the MTRR AA/AG genotype with clinical outcome that deserves further study in a larger patient cohort. A larger study would also give the opportunity to analyze how interactions between the MTHFR C677T, MTR A2756G and MTRR A66G variants may affect $p 16$ hypermethylation. In addition, there are other rare MTHFR, MTR and MTRR polymorphisms (9) that would be of interest to analyze in an extended study. The MTHFR A1298C variant, for instance, may be a possible risk factor for CRC, although the results published so far are highly controversial (10,30,54-56). The evaluation of the polymorphism as a predictor of clinical response to 5-FU-based chemotherapy has also led to inconclusive results $(10,18,57,58)$. Because of these inconsistent results, the polymorphism was not analyzed in the present study.

Some of the discrepancies that have been noted regarding the effects of genotypes are probably caused by differences among study populations relating to the average intake of dietary factors. Interactions between genotype and folate may only be detected in patients with high or low plasma folate levels $(11,12,55)$ or in patients receiving folate supplements (30). Thus, the lack of analysis of micronutrients in the tissue samples is a limitation of the present study. However, large populations are required to examine gene-nutrient interactions using traditional epidemiological methods.

In conclusion, our results indicate that patients with the MTRR 66 AA/AG genotypes have significantly worse cancer-specific survival when the mucosa is positive for $p 16$ hypermethylation. The reason for the particularly unfavorable outcome of patients having the combination of the MTRR 66 AA/AG genotype and $p 16$ hypermethylation in mucosa might be linked to a state of oxidative stress, which seems to be associated with this specific genotype as well as with $p 16$ methylation. Further studies are needed in order to analyze whether oxidative stress in macroscopically normal-appearing mucosa of patients with CRC leads to low folate/vitamin $\mathrm{B}_{12}$ levels and aberrant DNA methylation that could increase the risk of recurrent disease as well as a worse cancer-specific survival.

\section{ACKNOWLEDGMENTS}

We acknowledge the technical skills of M Martinsson, M Åkerström and J Flach. We thank H Björkqvist and A-L Helminen for collecting surgical tissue samples and L Munro, B Sjöberg and M-L Jakobsson for their work with the clinical database. We also thank the staff at the Genomics Core Facility in Gothenburg who performed the genotype analyses.

This work was supported by grants from the Swedish Cancer Society, the University of Gothenburg Foundation, the Assar Gabrielsson Foundation for Cancer Research, the Gustaf V Jubilee Clinic Foundation for Cancer Research and the Ingabritt and Arne Lundberg Foundation.

\section{DISCLOSURE}

The authors declare that they have no competing interests as defined by Molecular Medicine, or other interests that might be perceived to influence the results and discussion reported in this paper.

\section{REFERENCES}

1. Sharpless NE. (2005) INK4a/ARF: a multifunctional tumor suppressor locus. Mutat. Res. 576:22-38.

2. Alhaja E, et al. (2004) Anti-migratory and antiangiogenic effect of p16: a novel localization at membrane ruffles and lamellipodia in endothelial cells. Angiogenesis. 7:323-33.

3. Wettergren Y, Odin E, Nilsson S, Carlsson G, Gustavsson B. (2008) p16INK4a gene promoter hypermethylation in mucosa as a prognostic factor for patients with colorectal cancer. Mol. Med. 14:412-21.

4. Ulrich CM, et al. (2005) MTHFR variants reduce the risk of $\mathrm{G}: \mathrm{C}->\mathrm{A}: \mathrm{T}$ transition mutations within the p53 tumor suppressor gene in colon tumors. J. Nutr. 135:2462-7.

5. Oyama K, Kawakami K, Maeda K, Ishiguro K, Watanabe G. (2004) The association between methylenetetrahydrofolate reductase polymorphism and promoter methylation in proximal colon cancer. Anticancer Res. 24:649-54.

6. Mato JM, Lu SC. (2005) Homocysteine, the bad thiol. Hepatology. 41:976-9.

7. Peyrin-Biroulet L, et al. (2007) Vascular and cellu- 
lar stress in inflammatory bowel disease: revisiting the role of homocysteine. Am. J. Gastroenterol. 102:1108-15.

8. Toffoli G, et al. (2003) Methylenetetrahydrofolate reductase $677 \mathrm{C} \rightarrow \mathrm{T}$ polymorphism and risk of proximal colon cancer in north Italy. Clin. Cancer Res. 9:743-8.

9. Koushik A, et al. (2006) Nonsynonymous polymorphisms in genes in the one-carbon metabolism pathway and associations with colorectal cancer. Cancer Epidemiol. Biomarkers Prev. 15:2408-17.

10. Fernandez-Peralta AM, et al. (2010) Association of polymorphisms MTHFR C677T and A1298C with risk of colorectal cancer, genetic and epigenetic characteristic of tumors, and response to chemotherapy. Int. J. Colorectal Dis. 25:141-51.

11. Marugame T, et al. (2003) Relation of plasma folate and methylenetetrahydrofolate reductase C677T polymorphism to colorectal adenomas. Int. J. Epidemiol. 32:64-6.

12. Sharp L, Little J. (2004) Polymorphisms in genes involved in folate metabolism and colorectal neoplasia: a HuGE review. Am. J. Epidemiol. 159:423-43.

13. Keku T, et al. (2002) 5,10-Methylenetetrahydrofolate reductase codon 677 and 1298 polymorphisms and colon cancer in African Americans and whites. Cancer Epidemiol. Biomarkers Prev. 11:1611-21.

14. Shannon B, Gnanasampanthan S, Beilby J, Iacopetta B. (2002) A polymorphism in the methylenetetrahydrofolate reductase gene predisposes to colorectal cancers with microsatellite instability. Gut. 50:520-4.

15. Plaschke J, Schwanebeck U, Pistorius S, Saeger HD, Schackert HK. (2003) Methylenetetrahydrofolate reductase polymorphisms and risk of sporadic and hereditary colorectal cancer with or without microsatellite instability. Cancer Lett. 191:179-85.

16. Lima CS, et al. (2007) Polymorphisms in methylenetetrahydrofolate reductase gene (MTHFR) and the age of onset of sporadic colorectal adenocarcinoma. Int. J. Colorectal. Dis. 22:757-63.

17. Cohen V, et al. (2003) Methylenetetrahydrofolate reductase polymorphism in advanced colorectal cancer: a novel genomic predictor of clinical response to fluoropyrimidine-based chemotherapy. Clin. Cancer Res. 9:1611-5.

18. Etienne MC, et al. (2004) Methylenetetrahydrofolate reductase gene polymorphisms and response to fluorouracil-based treatment in advanced colorectal cancer patients. Pharmacogenetics. 14:785-792.

19. Derwinger K, Wettergren Y, Odin E, Carlsson G, Gustavsson B. (2009) A study of the MTHFR gene polymorphism C677T in colorectal cancer. Clin. Colorectal Cancer. 8, 43-8.

20. Chua W, et al. (2009) Molecular markers of response and toxicity to FOLFOX chemotherapy in metastatic colorectal cancer. Br. J. Cancer. 101:998-1004.

21. van der Put NM, et al. (1997) Sequence analysis of the coding region of human methionine synthase: relevance to hyperhomocysteinaemia in neural-tube defects and vascular disease. QJM. 90:511-7.

22. Leclerc D, et al. (1996) Human methionine synthase: cDNA cloning and identification of mutations in patients of the cblG complementation group of folate/cobalamin disorders. Hum. Mol. Genet. 5:1867-74.

23. Harmon DL, et al. (1999) Methionine synthase D919G polymorphism is a significant but modest determinant of circulating homocysteine concentrations. Genet. Epidemiol. 17:298-309.

24. Paz MF, et al. (2002) Germ-line variants in methyl-group metabolism genes and susceptibility to DNA methylation in normal tissues and human primary tumors. Cancer Res. 62:4519-24.

25. Kang S, et al. (2005) Polymorphism in folate- and methionine-metabolizing enzyme and aberrant CpG island hypermethylation in uterine cervical cancer. Gynecol. Oncol. 96:173-80.

26. Zijno A, et al. (2003) Folate status, metabolic genotype, and biomarkers of genotoxicity in healthy subjects. Carcinogenesis. 24:1097-103.

27. Wilson A, et al. (1999) A common variant in methionine synthase reductase combined with low cobalamin (vitamin B12) increases risk for spina bifida. Mol. Genet. Metab. 67:317-23.

28. Olteanu H, Munson T, Banerjee R. (2002) Differences in the efficiency of reductive activation of methionine synthase and exogenous electron acceptors between the common polymorphic variants of human methionine synthase reductase. Biochemistry. 41:13378-85.

29. de Vogel S, et al. (2009) Genetic variants of methyl metabolizing enzymes and epigenetic regulators: associations with promoter $\mathrm{CpG}$ island hypermethylation in colorectal cancer. Cancer Epidemiol. Biomarkers Prev. 18:3086-96.

30. Hubner RA, et al. (2006) Folate metabolism polymorphisms influence risk of colorectal adenoma recurrence. Cancer Epidemiol. Biomarkers Prev. 15:1607-13

31. Yamaji T, et al. (2009) Methionine synthase A2756G polymorphism interacts with alcohol and folate intake to influence the risk of colorectal adenoma. Cancer Epidemiol. Biomarkers Prev. 18:267-74.

32. Kraunz KS, et al. (2006) Dietary folate is associated with p16(INK4A) methylation in head and neck squamous cell carcinoma. Int. J. Cancer. 119:1553-7.

33. Kamiya H, et al. (1998) A methylenetetrahydrofolate reductase polymorphism is associated with expression of p16 in human lung cancer. Oncol. Rep. 5:911-4.

34. Hamilton SR, Aaltonen LA. (2000) Pathology and Genetics of Tumours of the Digestive System. Lyon: IARC press. $314 \mathrm{pp}$.

35. Compton CC, et al. (2000) Prognostic factors in colorectal cancer. College of American Pathologists Consensus Statement 1999. Arch. Pathol. Lab. Med. 124:979-94.

36. Herman JG, et al. (1995) Inactivation of the CDKN2/p16/MTS1 gene is frequently associated with aberrant DNA methylation in all common human cancers. Cancer Res. 55:4525-30.

37. Belshaw NJ, et al. (2008) Profiling CpG island field methylation in both morphologically normal and neoplastic human colonic mucosa. $\mathrm{Br}$. J. Cancer. 99:136-42.

38. Chen LC, et al. (2004) Alteration of gene expression in normal-appearing colon mucosa of $\mathrm{APC}(\mathrm{min})$ mice and human cancer patients. Cancer Res. 64:3694-700.

39. Gloria L, et al. (1996) DNA hypomethylation and proliferative activity are increased in the rectal mucosa of patients with long-standing ulcerative colitis. Cancer. 78:2300-6.

40. Franco R, Schoneveld O, Georgakilas AG, Panayiotidis MI. (2008) Oxidative stress, DNA methylation and carcinogenesis. Cancer Lett. 266:6-11.

41. Tanaka T, Iwasa Y, Kondo S, Hiai H, Toyokuni S. (1999) High incidence of allelic loss on chromosome 5 and inactivation of p15INK4B and p16INK4A tumor suppressor genes in oxystressinduced renal cell carcinoma of rats. Oncogene. 18:3793-7.

42. Toyokuni S. (2008) Molecular mechanisms of oxidative stress-induced carcinogenesis: from epidemiology to oxygenomics. IUBMB Life. 60:441-7.

43. Romanenko A, et al. (2002) P16INK4A and p15INK4B gene alteration associated with oxidative stress in renal cell carcinomas after the chernobyl accident (pilot study). Diagn. Mol. Pathol. 11:163-9.

44. Belinsky SA, et al. (2002) Aberrant CpG island methylation of the p16(INK4a) and estrogen receptor genes in rat lung tumors induced by particulate carcinogens. Carcinogenesis. 23:335-9.

45. Govindarajan B, et al. (2002) Reactive oxygen-induced carcinogenesis causes hypermethylation of p16(Ink4a) and activation of MAP kinase. Mol. Med. 8:1-8.

46. Jang TJ, Kim DI, Shin YM, Chang HK, Yang CH. (2001) p16(INK4a) promoter hypermethylation of non-tumorous tissue adjacent to gastric cancer is correlated with glandular atrophy and chronic inflammation. Int. J. Cancer. 93:629-34.

47. O’Byrne KJ, Dalgleish AG. (2001) Chronic immune activation and inflammation as the cause of malignancy. Br. J. Cancer. 85:473-83.

48. van Engeland M, et al. (2003) Effects of dietary folate and alcohol intake on promoter methylation in sporadic colorectal cancer: the Netherlands cohort study on diet and cancer. Cancer Res. 63:3133-7.

49. Tchantchou F. (2006) Homocysteine metabolism and various consequences of folate deficiency. J. Alzheimers Dis. 9:421-7.

50. Rogers EJ, Chen S, Chan A. (2007) Folate deficiency and plasma homocysteine during increased oxidative stress. N. Engl. J. Med. 357:421-2.

51. Morgenstern I, Raijmakers MT, Peters WH, Hoensch H, Kirch W. (2003) Homocysteine, cysteine, and glutathione in human colonic mucosa: elevated levels of homocysteine in patients with 
inflammatory bowel disease. Dig. Dis. Sci. 48:2083-90.

52. Danese $S$, et al. (2005) Homocysteine triggers mucosal microvascular activation in inflammatory bowel disease. Am. J. Gastroenterol. 100:886-95.

53. Peyrin-Biroulet L, et al. (2008) Association of MTRR $66 \mathrm{~A}>\mathrm{G}$ polymorphism with superoxide dismutase and disease activity in patients with Crohn's disease. Am. J. Gastroenterol. 103:399-406.

54. Chen J, et al. (2002) Linkage disequilibrium between the $677 \mathrm{C}>\mathrm{T}$ and $1298 \mathrm{~A}>\mathrm{C}$ polymorphisms in human methylenetetrahydrofolate reductase gene and their contributions to risk of colorectal cancer. Pharmacogenetics. 12:339-42.

55. Giovannucci E, et al. (2003) Methylenetetrahydrofolate reductase, alcohol dehydrogenase, diet, and risk of colorectal adenomas. Cancer Epidemiol. Biomarkers Prev. 12:970-9.

56. Curtin K, et al. (2004) MTHFR C677T and A1298C polymorphisms: diet, estrogen, and risk of colon cancer. Cancer Epidemiol. Biomarkers Prev. 13:285-92.

57. Marcuello E, Altes A, Menoyo A, Rio ED, Baiget M. (2006) Methylenetetrahydrofolate reductase gene polymorphisms: genomic predictors of clinical response to fluoropyrimidine-based chemotherapy? Cancer Chemother. Pharmacol. 57:835-40.

58. Zintzaras E, et al. (2009) MTHFR gene polymorphisms and response to chemotherapy in colorectal cancer: a meta-analysis. Pharmacogenomics. 10:1285-94. 